\title{
Hazard Assessment of Air Pollutants: The Transforming Ability of Complex Pollutant Mixtures in the Bhas 42 Cell Model
}

\author{
Stefania Serral, Monica Vaccari², Maria Grazia Mascolo², Francesca Rotondo², Cristina Zanzi², \\ Laura Polacchini 1, Christine Behle Wagner ${ }^{3}$, Thorsten Kunkelmann ${ }^{3}$, Sabine Perschbacher ${ }^{3}$, Albrecht Poth ${ }^{4}$, \\ Sandro Grilli' ${ }^{1}$, Miriam N. Jacobs 5 and Annamaria Colacci ${ }^{2}$ \\ ${ }^{1}$ Department of Experimental, Diagnostic and Specialty Medicine-Cancer Research Section, University of Bologna, Bologna, Italy; ${ }^{2}$ Center for \\ Environmental Toxicology and Risk Assessment, Regional Agency for Prevention, Environment and Energy Emilia Romagna Region (Arpae), \\ Bologna, Italy; ${ }^{3}$ ENVIGO CRS GmbH, Rossdorf, Germany; ${ }^{4}$ Dr. Knoell Consult GmbH, Mannheim, Germany; ${ }^{5}$ Department of Toxicology, Centre \\ for Radiation, Chemical and Environmental Hazards Public Health England, Chilton, Oxfordshire, UK
}

\begin{abstract}
The use of in vitro alternative methods is a promising approach to characterize the hazardous properties of environmental chemical mixtures, including urban airborne particulate matter (PM). The aim of this study was to examine seasonal differences in the toxic and transforming potential of PM samples by using the in vitro cell transformation assay in Bhas 42 cells for the prediction of potential carcinogenic effects. Bhas 42 cells are already initiated, and the $v$-Ha-ras transfection, together with genetic modification following the immortalization process, makes them a valuable model to study the late steps of cellular transformation leading to the acquisition of the malignant phenotype. Exposure to organic extracts of $\mathrm{PM}_{1}$ and $\mathrm{PM}_{2.5}$ induced dose-related effects. The transforming and cytotoxic properties were related to the amount of PM collected during the sampling campaign and associated with the concentrations of polycyclic aromatic hydrocarbons (PAHs) in the samples. All the samples induced cell transformation following prolonged exposure over 2 weeks. Our results support the utility of the in vitro top-down approach to characterize the toxicity of real mixtures, thereby supporting regulators in the decision-making process. The results also identify the need for appropriate assay selection within the in vitro testing strategy to address the complexity of adverse outcomes.
\end{abstract}

\section{Introduction}

Air pollution is known to increase the risk for a wide range of adverse health outcomes (Landrigan et al., 2018; WHO, 2016). Studies indicate that exposure levels have increased in recent years, particularly in rapidly industrializing countries (Landrigan et al., 2018). Management of risks associated with air pollution, however, remains challenging, even in countries where advanced technologies and environmental policies are in place (Colacci and Vaccari, 2017).

In 2013, the International Agency for Research on Cancer (IARC) classified outdoor air pollution and particulate matter (PM) from outdoor air pollution as carcinogenic to humans (Group 1) (Loomis et al., 2013; IARC, 2016). This evaluation is based on sufficient evidence of carcinogenicity in humans and ex- perimental animals (IARC, 2016). Epidemiological studies have demonstrated a clear association between lifetime exposure to PM and lung cancer (Gharibvand et al., 2017; Hamra et al., 2014). This evidence is supported by strong mechanistic data, demonstrating the induction of mutagenic and genotoxic effects in humans and experimental systems (IARC, 2016). Moreover, PM also can play a role in the non-genotoxic origin of cancer, through the induction of oxidative stress-sustained inflammation (IARC, 2016).

Difficulties in the risk assessment of human exposure to air pollution arise due to knowledge gaps in identifying multiple components of complex mixtures, the lack of toxicological information regarding their carcinogenic potential, and the limited approach to cumulative risk assessment from multiple exposures via multiple routes.
Received December 17, 2018; Accepted May 10, 2019

Epub June 3, 2019; ( ) The Authors, 2019.

ALTEX 36(4), 623-633. doi:10.14573/altex.1812173

Correspondence: Annamaria Colacci, PhD

Center for Environmental Toxicology and Risk Assessment

Regional Agency for Prevention,

Environment and Energy Emilia Romagna Region (Arpae),

viale Filopanti 22, 40126 Bologna, Italy

(annamaria.colacci@unibo.it)
This is an Open Access article distributed under the terms of the Creative Commons Attribution 4.0 International license (http://creativecommons.org/licenses/by/4.0/) which permits unrestricted use, distribution and reproduction in any medium, provided the original work is appropriately cited. 
Environmental samples are characterized by the simultaneous presence of a large number of pollutants, showing different mechanisms of action and toxicity profiles. The concentration of the single components in the mixture varies according to the emission sources and the site of sampling. Besides carcinogenic chemicals, mixtures may contain chemicals that are not classified as carcinogens but affect biological pathways relevant to the carcinogenesis process, resulting in carcinogenic synergies (Goodson et al., 2015).

In the global regulatory context, the carcinogenic potential of chemicals is conventionally explored using the 2 -year rodent carcinogenicity bioassay (RCB) in an approach that is usually limited to single chemicals, particularly pesticides. Other industrial chemicals are rarely tested in the RCB (Jacobs et al., 2016). Currently, several in vitro tests addressing key events related to the carcinogenesis process are at different stages of development (validation) and regulatory acceptance. To date, however, they can only partially replace animal tests used to evaluate the hazard of substances (Corvi et al., 2017). Whilst the Organisation for Economic Co-operation and Development (OECD) Test Guideline Programme has agreed that validated in vitro test methods can be used for chemical mixtures, such mixtures are generally not used in validation exercises.

The in vitro cell transformation assay (CTA) appears to be one of the most promising approaches to predict the carcinogenic hazard of chemicals, complex mixtures, and environmental pollutants (Lilienblum et al., 2008; Mascolo et al., 2010; Corvi and Vanparys, 2012; Vasseur and Lasne, 2012; Corvi et al., 2017). The CTA is one of the accepted methods for the evaluation of toxicological properties of chemicals under the Registration, Evaluation, Authorisation and Restriction of Chemical Substances (REACH) Regulation (EU, 2008). It measures the morphological transformation of cells as transformed colonies or foci derived from a single cell. It is understood to involve a multistage process that closely models some key stages of in vivo carcinogenesis (Vanparys et al., 2012; Vasseur and Lasne, 2012).

Various types of CTAs have been developed and are in use. The Syrian hamster embryo (SHE) CTA uses primary cells. Other common CTAs, such as C3H10T1/2, BALB/c 3T3, and Bhas 42 CTAs, are based on the use of established cell lines. The international validation studies carried out on CTAs suggest that these assays can be considered as scientifically valid for assessing the carcinogenic potential of hazardous compounds (Corvi et al., 2012; Sakai et al., 2011) and may provide suitable alternatives to the RCB when used in conjunction with other test methods (Vanparys et al., 2011).

In 2007, the OECD proposed to use the CTA as a second-level screening tool for carcinogens and as a screening test of choice for non-genotoxic carcinogens, which are not detected in the standard regulatory "mutagenicity assays" (UN, 2017; OECD, 2017). Whilst it has since been concluded by the OECD that the CTA is not suitable as a stand-alone assay to predict carcinogenesis, it can be utilized together with other relevant in vitro assays and experimental results, also including genotoxicity data, structure-activity analysis, and pharmaco-toxicokinetic information, as part of a testing strategy and/or in a weight-of-evidence mode of action approach, particularly for the endpoint related to morphological transformation (Jacobs et al., 2016).

Among the CTA models, the Bhas 42 CTA has been proposed as a sensitive, short-term system for predicting chemical carcinogenicity. Bhas 42 cells were derived from the mouse embryo fibroblast cell line BALB/c 3T3 A31-1-1 by transfection with a plasmid containing the $v$-Ha-ras gene (Sasaki et al., 1988, 1990a; Uchio-Yamada et al., 2017). Bhas 42 cells have recently been correctly identified as originating from Swiss mouse cells (Sasaki et al., 1988; Uchio-Yamada et al., 2017; OECD, 2017). After the $v$-Ha-Ras transfection, these cells retain some characteristics of the original clone, such as the sensitivity to contact inhibition and the susceptibility to chemically-induced transformation. After exposure to carcinogenic agents, they form transformed foci, arising from morphologically altered cells, which acquire the ability to invade the surrounding non-transformed contact-inhibited monolayer (Sasaki et al., 1988). Untransformed Bhas-42 cells are not tumorigenic upon transplantation in vivo and grow to confluence, forming a contact-inhibited monolayer, in vitro.

Since Bhas 42 cells are transfected with and express the activated $v$-Ha-ras oncogene, they are regarded as already initiated cells, according to the two-stage paradigm of genotoxic carcinogenesis (Sasaki et al., 1990a, 2015). However, the proposed Bhas 42 CTA protocol includes both an "initiation" assay and a "promotion" assay, which can be performed independently (Sakai et al., 2010, 2011). The main differences between the two assays are the number of plated cells and the treatment schedule (Tab. S1 ${ }^{1}$ ). In the "initiation" assay, the cells are inoculated at a low density and treated with the test chemical for a short time period (from Day 1 to 4 after plating) at the beginning of the growth phase (acute exposure). During this period, the cells undergo several cell divisions before reaching confluence, increasing the likelihood that any DNA damage would escape repair and become fixed within the cell. It is also possible that cell division can increase transcription-coupled repair, which may not be active in quiescent cells. In the "promotion" assay, the cells are seeded at a higher density than in the "initiation" assay; the treatment is started at sub-confluence and continued during the stationary phase for a longer time period (from Day 4 to 14 after plating), mimicking a late and repeated exposure. Thus, the Bhas-42 "initiation/promotion assay" does not completely reflect the initiation/promotion stages in the multistep process of human carcinogenesis or the initiation/promotion scheme in the experimental animal models. However, this is how the Bhas 42 "initiation/promotion assay" is

\footnotetext{
1 doi:10.14573/altex.1812173s
}

\section{Abbreviations}

ACE, absolute clonal efficiency; CLP, Classification, Labelling and Packaging; CTA, cell transformation assay; CV, crystal violet; DMSO, dimethylsulfoxide; FBS, fetal bovine serum; IARC, International Agency for Research on Cancer; IATA, Integrated Approach to Testing and Assessment; 3-MCA, 3-methylcholanthrene; MEM, minimum essential medium; NPAH, nitro-PAH; OD, optical density; OECD, Organisation for Economic Co-operation and Development; PAH, polycyclic aromatic hydrocarbons; PM, particulate matter; RCB, rodent carcinogenicity bioassay; RCE, relative clonal efficiency; REACH, Registration, Evaluation, Authorization, and Restriction of Chemicals; SHE, Syrian hamster embryo; TPA, tetradecanoylphorbol-13-acetate; UVCBs, Substances of unknown or variable composition, complex reaction products, or biological materials 
incorrectly described in the Bhas 42 CTA OECD Guidance Document (OECD, 2017). Therefore, we will use the more appropriate descriptive terms "acute exposure assay" and "late repeated exposure assay" instead of "initiation assay" or "promotion assay" throughout this paper.

Although the Bhas 42 CTA was validated using single chemicals (Sakai et al., 2011), the test method is technically applicable to mixtures and has been used to investigate the transforming effects of cigarette smoke condensate (Weisensee et al., 2013; Han et al., 2016) and PM extracts (Ohmori et al., 2013). Indeed, the original source of Bhas 42 cells, BALB/c A31-1-1, can sustain the metabolism of PAH to carcinogenic intermediates (Kakunaga and Crow, 1980; Lo and Kakunaga, 1982).

In the present study, the Bhas 42 CTA was performed to highlight the toxic and carcinogenic potential of urban airborne particulate matter samples $\left(\mathrm{PM}_{1}\right.$ and $\left.\mathrm{PM}_{2.5}\right)$ collected during different seasons at a site located in the north of Bologna, Italy. The PM organic extracts were analyzed with the aim of confirming the suitability of the Bhas 42 CTA for testing complex mixtures.

\section{Material and methods}

\subsection{Cells}

The Bhas 42 cell line was purchased from the Hatano Research Institute (HRI) (Hadano, Japan). Cells were expanded in Minimum Essential Medium (MEM) supplemented with 10\% fetal bovine serum (FBS) and 1\% 100x penicillin/streptomycin solution (M10F), and cryopreserved in MEM 10\% FBS solution containing 5\% dimethylsulfoxide (DMSO). Cells at passage 2 after thawing were used for the CTAs. Frozen Bhas 42 cells were rapidly thawed and cultured in M10F up to about $70 \%$ confluence and then cultured in Dulbecco's modified Eagle's medium/Ham's F12 supplemented with 100 units/ml of penicillin, $100 \mu \mathrm{g} / \mathrm{ml}$ of streptomycin, and 5\% FBS (DF5F) to let cells reach about 70\% confluence again, before seeding them for the CTA.

\subsection{Collection, preparation, and characterization of PM samples}

The $\mathrm{PM}_{2.5}$ fraction was collected using the high-volume air flow sampler Air Flow PM 2.5 -HVS (UNI-EN 14907 compliant, suction flow 500 1/min, Air Monitoring Systems-Analitica, Pesaro, Italy). The $\mathrm{PM}_{1}$ samples were collected using Air Flow poly- urethane foam (PUF) (suction flow 200 1/min, Air Monitoring Systems-Analitica, Pesaro, Italy), which could simultaneously collect the gas fraction (using PUF-polyurethane foam). Each filter was weighed daily before and after PM collection, in order to obtain gravimetric data. All filters from each season were pooled to obtain a single sample that was representative of the season. In order to obtain the organic fraction, each pooled sample was extracted with acetone using a Soxhlet apparatus. It was then dried and dissolved in sterile DMSO (CAS number 67-685) at $800 \mathrm{~m}^{3}$ equivalents $/ \mathrm{ml}$.

Aliquots of the organic extracts were analyzed as previously described (Bocchi et al., 2016). Briefly, acetone extracts were purified by solvent elution using solid-phase extraction cartridges packed with silica gel adsorbent. PAHs and nitro-PAHs (NPAHs) eluted together in the dichloromethane/hexane (1:1) fraction. PAHs were identified and quantified by the Thermo Scientific DFS $^{\text {TM }}$ Magnetic Sector GC-HRMS system. Deuterated PAH standards were used for quantification. Measurements of NPAHs were carried out by high resolution gas chromatography interfaced to a mass spectrometer triple quadrupole at low resolution (HRGC/GC/MS/MS). The quantitative analysis was performed on the basis of an external calibration using a mixture of reference standards.

A brief description of the PM samples is reported in Table 1. The complete list of the compounds detected and measured in the analyzed extracts is reported in Table S2 ${ }^{1}$.

\subsection{Rationale for dose selection and dosing regime}

$\mathrm{PM}_{1}$ and $\mathrm{PM}_{2.5}$ samples were collected at an urban site in Region Emilia Romagna, Italy (WGS84, longitude 44.524, latitude 11.34) during two different monitoring campaigns performed in autumn (October 23 - November 12, 2012) and summer (May 6 - May 28, 2013). The PM organic extracts were tested in the Bhas 42 CTA according to the validated protocol (OECD, 2017). The experimental design is sketched in Figure 1.

Due to the limited amount of PM extracts, dose-range finding cytotoxicity assays were not performed, but prior experimental knowledge was used to guide dose selection (Vaccari et al., 2015). Cells were treated with concentrations selected to correspond to a volume of air ranging from $2 \mathrm{~m}^{3}$ to $8 \mathrm{~m}^{3}$, as this is representative of realistic exposures during human outside activity, calculated on the basis of standard US Environmental Protection Agency guidance regarding the daily average inhalation

Tab. 1: Description of PM samples

PAHs were identified and quantified by the Thermo Scientific DFS TM Magnetic Sector GC-HRMS system. Deuterated PAH standards were used for quantification. The measurements of NPAHs were carried out by high resolution gas chromatography interfaced to a mass spectrometer triple quadrupole at low resolution (HRGC/GC/MS/MS). The quantitative analysis was performed with an external calibration, using a mixture of reference standards.

\begin{tabular}{|l|l|l|l|l|}
\cline { 2 - 5 } \multicolumn{1}{c|}{} & \multicolumn{2}{l|}{ Autumn 2012} & \multicolumn{2}{l|}{ Summer 2013 } \\
\cline { 2 - 5 } \multicolumn{1}{c|}{} & $\mathrm{PM}_{\mathbf{1}}$ & $\mathrm{PM}_{\mathbf{2 . 5}}$ & $\mathrm{PM}_{\mathbf{1}}$ & $\mathrm{PM}_{\mathbf{2 . 5}}$ \\
\hline $\mathrm{PM}\left[\mu \mathrm{g} / \mathrm{m}^{3}\right]$ & 19.86 & 27.27 & 7.24 & 9.47 \\
\hline $\boldsymbol{\Sigma} \mathrm{PAH}+\boldsymbol{\Sigma} \mathrm{NPAH}^{*}\left[\mathrm{ng} / \mathrm{m}^{3}\right]$ & 3.87 & 3.89 & 0.27 & 0.26 \\
\hline
\end{tabular}

* total amount of PAHs and nitro-PAHs measured in the PM organic extracts 


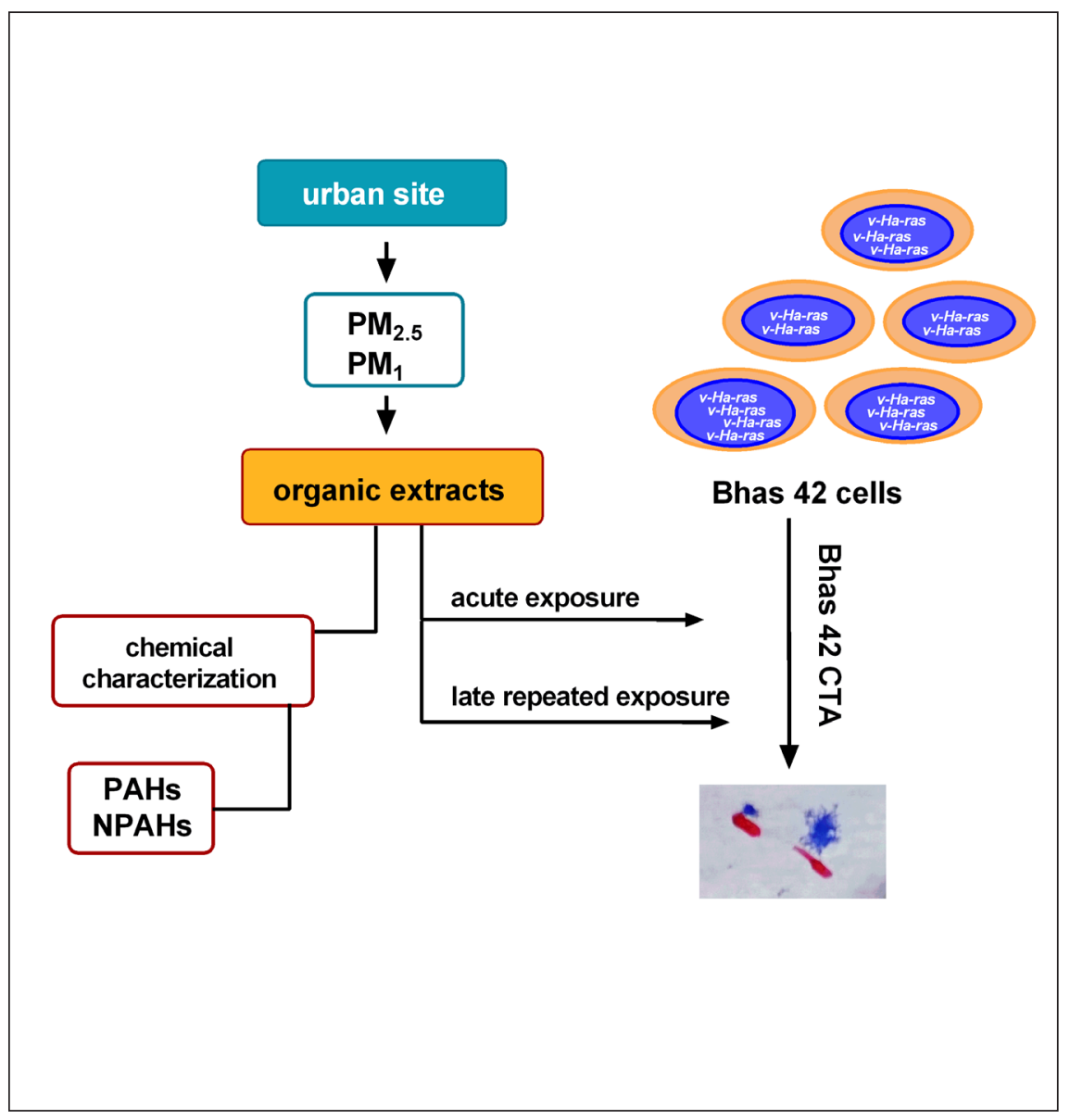

Fig. 1: Experimental design

$\mathrm{PM}_{1}$ and $\mathrm{PM}_{2.5}$ samples were collected at an urban site during two different campaigns performed in autumn 2012 and summer 2013. The $\mathrm{PM}_{2.5}$ fraction was collected by a high-volume air flow sampler, Air Flow $\mathrm{PM}_{2.5}$-HVS (UNI-EN 14907 compliant, suction flow $500 \mathrm{l} / \mathrm{min}$ ). The $\mathrm{PM}_{1}$ samples were collected by Air Flow polyurethane foam (PUF) (suction flow $200 \mathrm{l} / \mathrm{min}$ ), which could simultaneously collect the gas fraction (using PUF-polyurethane foam). Each filter was weighed daily before and after PM collection. All the filters from each season were pooled to obtain a single sample, which was representative of the season. In order to obtain the organic fraction, each pooled sample was extracted with acetone using a Soxhlet apparatus. It was then dried and dissolved in DMSO at $800 \mathrm{~m}^{3}$ equivalents/ml. Organic extracts were purified by solvent elution using solid-phase extraction cartridges packed with silica gel adsorbent. PAHs and nitro-PAHs (NPAHs) eluted together in the dichloromethane/ hexane (1:1) fraction. The analytes were identified and quantified. The PM organic extracts were tested in the Bhas 42 CTA. Cells were treated for $72 \mathrm{~h}$ (acute exposure) or 10 days (late repeated exposure).

rates for long-term exposure of the general population (US-EPA, 2011), where ranges are from maximal exposure (adults during $8 \mathrm{~h}$ of outside activity, $95 \%$ percentile) to minimal exposure (1-3 month old children). In order to highlight the seasonal differences between realistic exposures, the experimental assay treatments were carried out using equal volumetric concentrations of air $\left(\mathrm{m}^{3}\right)$ for all the samples, even if the amount of PM differed. The working solutions for the cell treatment were prepared by diluting the DMSO stock solutions in DF5F immediately before use, at final concentrations ranging from $2 \mathrm{~m}^{3}$ to $8 \mathrm{~m}^{3}$ equivalents/well in 6-well microplates (1-4 $\mathrm{m}^{3}$ equivalents/ml). The final concentration of the solvent vehicle DMSO was $0.5 \%$.

\subsection{Cell transformation assay}

Schedule 1 - Acute exposure assay

Cells were seeded at $4 \times 10^{3}$ cells/well (Day 0). Cell treatment started $24 \mathrm{~h}$ later (Day 1) and continued for $72 \mathrm{~h}$. DF5F medium was replaced on Days 4, 7, 10, and 14. On Day 21, cells were fixed with methanol and stained with 5\% Giemsa solution. Nine wells were prepared for each concentration, of which 6 wells were reserved for the transformation assay, and three for the cell growth assay. Negative controls were represented by DMSO-treated cells (vehicle/solvent controls). 3-Methylcholanthrene (3-MCA, CAS number 56-49-5, $1 \mu \mathrm{g} / \mathrm{ml}$ ) served as the positive control, as prescribed in the CTA protocol (OECD, 2017).
For cell growth evaluation, cells were fixed with $10 \%$ formalin and stained with $0.1 \%$ crystal violet on Day 7 . Then the dye was extracted and optical density (OD) was measured at 540-570 nm. The relative cell growth was calculated as relative cell growth $(\%)=[($ treatment - blank $) /($ control - blank $)] \times 100$.

\section{Schedule 2 - Late repeated exposure assay}

Cells were plated at a density of $14 \times 10^{3}$ cells/well and cultured for 4 days without replacing medium. On Days 4, 7, and 10, treatment solutions in DF5F were delivered. The treatment was continued until Day 14. Cells were then maintained in DF5F until Day 21. Solvent-treated controls were represented by DMSO-treated cells. TPA (tetradecanoylphorbol-13-acetate, CAS number 16561$29-8,50 \mathrm{ng} / \mathrm{ml}$ ) served as the positive control. The concurrent cell growth assay plates were fixed and stained on Day 7.

\section{Counting of transformed foci and statistical analysis}

Foci were classified as fully malignant type III foci when more than 100 cells contributed to the formation of the focus and cells showed the following morphological characteristics: (a) spindle-shaped morphology that is distinctly different from the contact-inhibited monolayer cells; (b) deeply basophilic staining; (c) random orientation; (d) dense multilayering; (e) invasion of the monolayer of surrounding contact-inhibited cells. The number of type III foci in each well was recorded. 


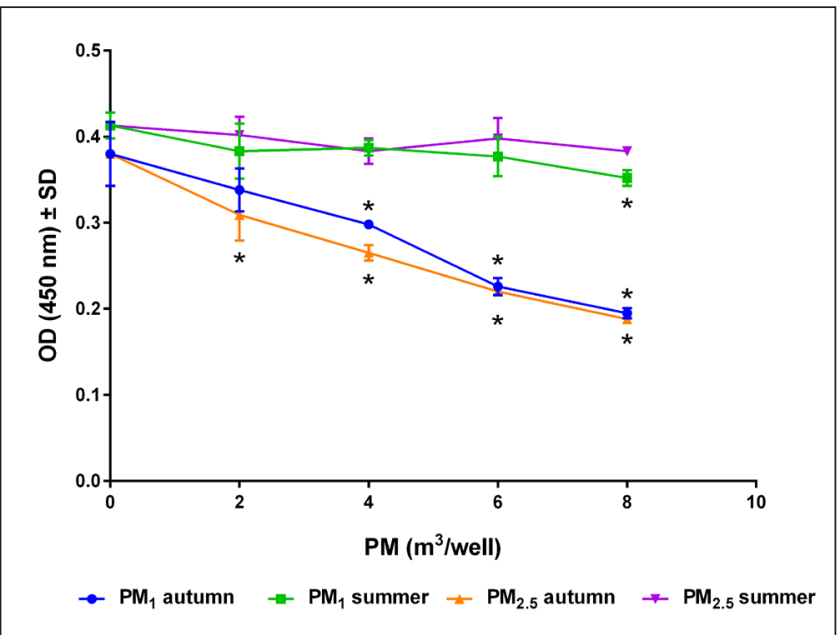

Fig. 2: Cytotoxic effects induced by organic extracts of $P M_{1}$ and $\mathrm{PM}_{2.5}$ ( $\mathrm{m}^{3} /$ well) in the acute exposure assay

Cells were seeded at $4 \times 10^{3}$ cells/well (Day 0), incubated for $24 \mathrm{~h}$, and then exposed to the $\mathrm{PM}_{2.5}$ and $\mathrm{PM}_{1}$ organic extracts at concentrations ranging from 2 to $8 \mathrm{~m}^{3}$ equivalent/well (1-4 m3/ml). Three wells were prepared for each treatment group. The exposure duration lasted $72 \mathrm{~h}$. On Day 4, the medium containing the test chemical was replaced with fresh DF5F medium. On Day 7 , cells were fixed with $10 \%$ formalin and stained with a $0.1 \%$ crystal violet solution for $15 \mathrm{~min}$. The dye was extracted from the stained cells with $2 \mathrm{ml}$ of dye extraction solution (containing $0.02 \mathrm{~mol} / \mathrm{l} \mathrm{HCl}$ and $50 \%$ ethanol). Cytotoxicity was determined by measuring the OD at $540-570 \mathrm{~nm}$. SD, standard deviation; ${ }^{*}, \mathrm{p}<0.05 \mathrm{vs}$ controls (solvent-treated cells); one-sided Dunnett test.
The statistical analysis was performed by multiple comparisons using the one-sided Dunnett test $(\mathrm{p}<0.05)$. The results from the Bhas 42 cell transformation assays were judged positive when there were two or more sequential concentrations that induced statistically significant increases in the number of transformed foci, and negative when there was no statistically significant increase of transformed foci at any of the tested concentrations.

\section{Results}

\subsection{Evaluation of cytotoxicity of $P M_{1}$ and $P M_{2.5}$}

The acute treatment with $\mathrm{PM}_{1}$ or $\mathrm{PM}_{2.5}$ organic extracts derived from the autumn campaign induced a concentration-related cytotoxic effect. The reduction in cell viability was about $50 \%$ at the highest concentrations $\left(6 \mathrm{~m}^{3} /\right.$ well and $8 \mathrm{~m}^{3} /$ well) (Fig. 2). The late repeated exposure assay was conducted only for the $\mathrm{PM}_{2.5}$ autumn extract. No cytotoxic effects were observed at the end of the treatment period, even at the highest applied concentration.

The lower content of $\mathrm{PM}_{1}$ and $\mathrm{PM}_{2.5}$ summer extracts (Tab. 1) did not induce cytotoxic effects in either the acute or the late repeated exposure assay (Tab. S3 ${ }^{1}$, Fig. 2).

The observed toxicity was related to the tested sample and was mainly associated with the season of sampling (Fig. 2). This association was confirmed by comparison of the results from the cell growth assay with the amount of particulate (PM, $\mu \mathrm{g} /$ well), or the total amount of PAHs and nitro-PAHs ( $\sum$ PAHs $+\sum$ NPAHs, $\mathrm{ng} /$ well) corresponding to the $\mathrm{m}^{3}$-equivalents present in the treatment solutions (Fig. 3).

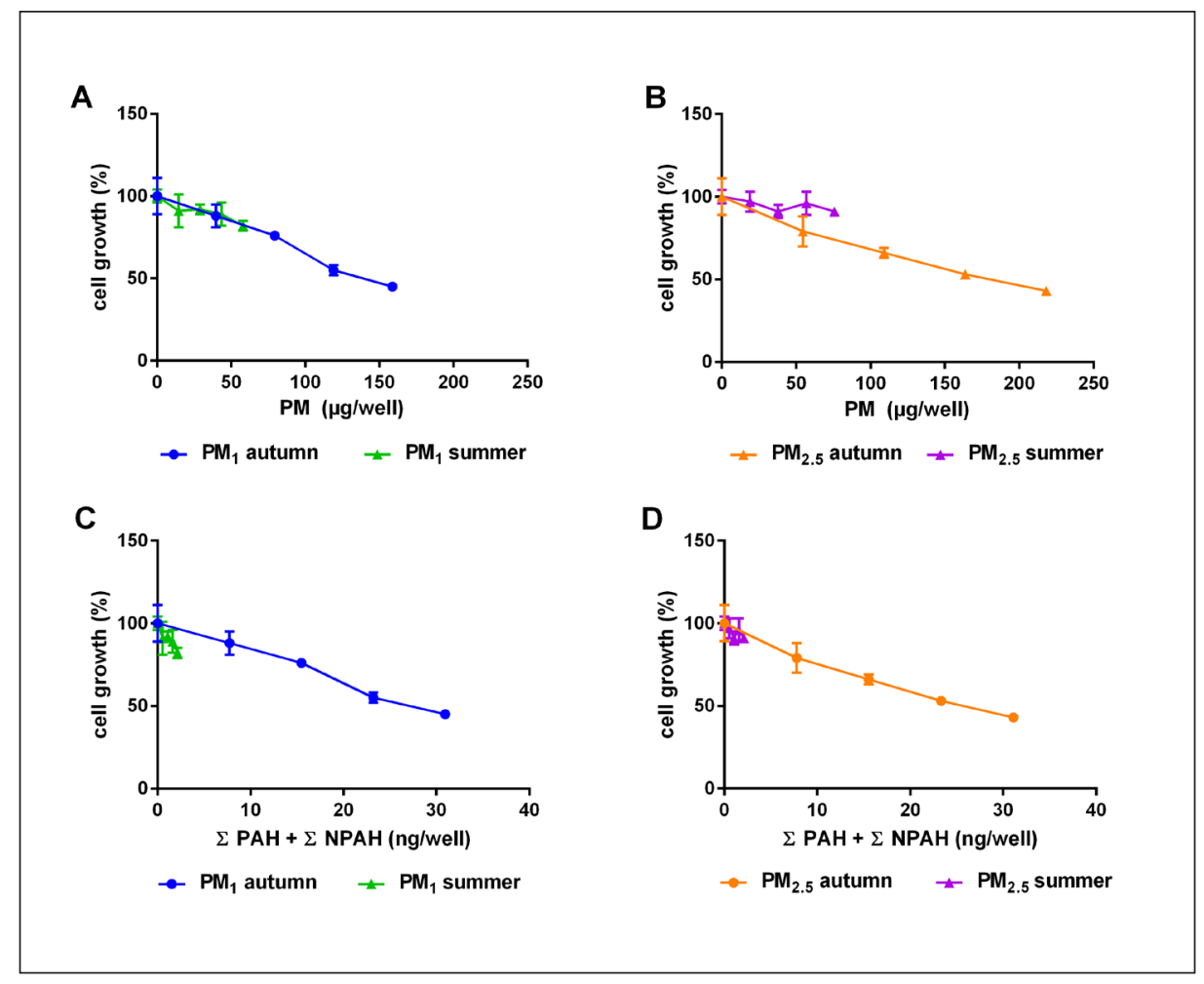

Fig. 3: Relationships between cytotoxic effects and the total amount of PM or PAHs and NPAHs present in each treatment extract Cells were treated with $2-8 \mathrm{~m}^{3} /$ well of PM extracts for $72 \mathrm{~h}$ (acute exposure assay, Schedule 1). A, B = PM ( $\mu \mathrm{g} /$ well); C, D $=\Sigma$ PAHs $+\Sigma$ NPAHs (ng/well); A, C = PM extracts; $\mathrm{B}, \mathrm{D}=\mathrm{PM}_{2.5}$ extracts. Data are expressed as cell growth (\% vs solvent control) $\pm \mathrm{SD}$. 


\section{Acute exposure assay}
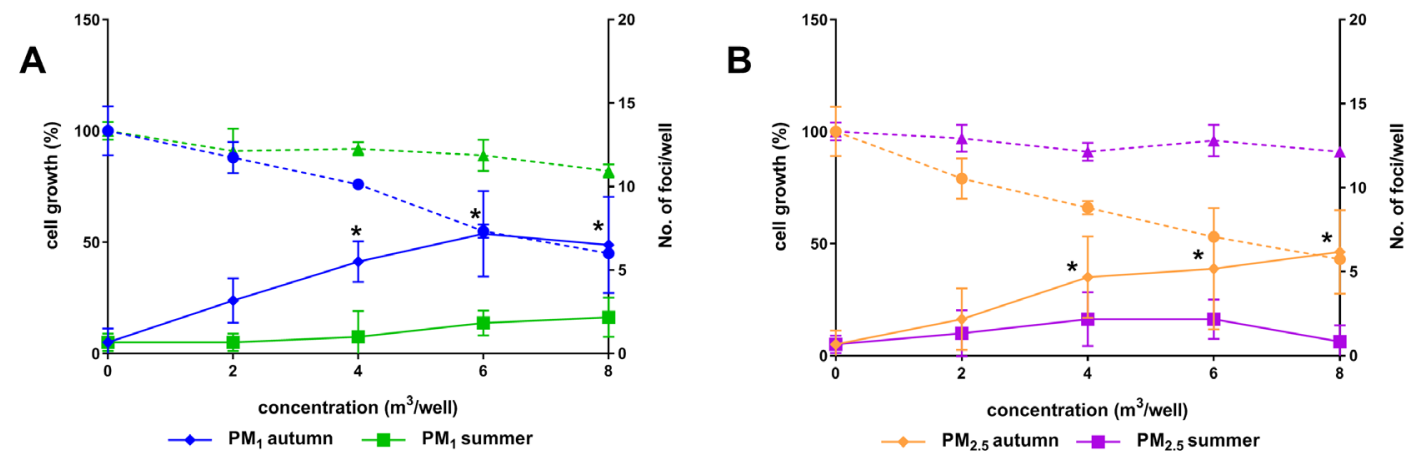

\section{Late repeated exposure assay}
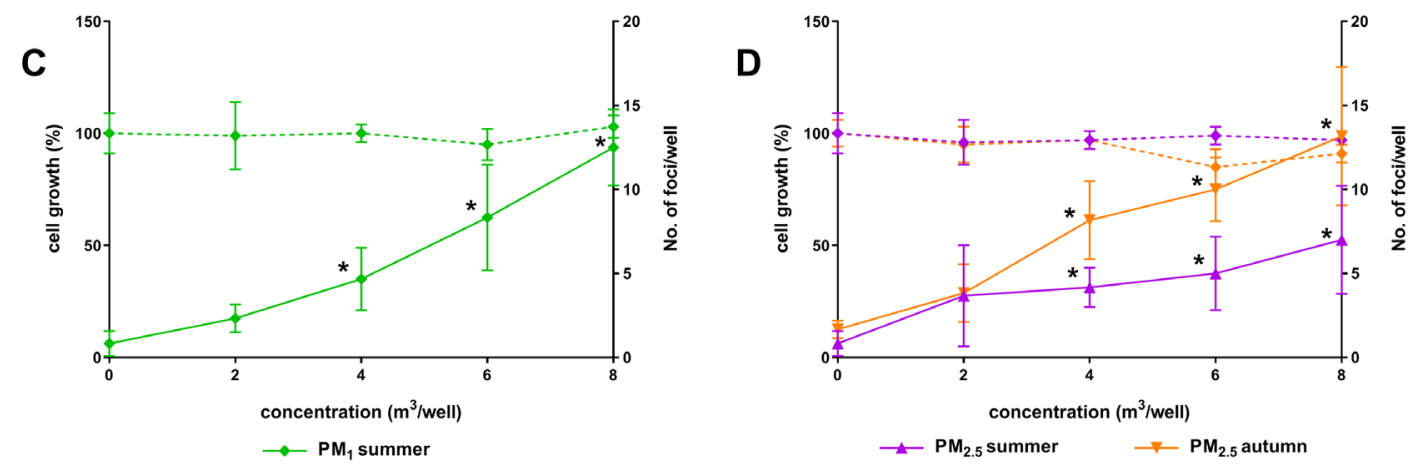

Fig. 4: Graphic representation of data from the transformation assays and the concurrent cell growth assays

In the acute exposure assays, the cells were inoculated at a low density $\left(4 \times 10^{3}\right.$ cells/well, Day 0$)$ and treated with the test chemical for a short period (from Day 1 to 4 after plating) at the beginning of the growth phase. In the late repeated exposure assays, the cells were seeded at high density (14 × $10^{3}$ cells/well, Day 0); the treatment is started at sub-confluence (Day 4) and continued during the stationary phase for a longer period (from Day 4 to 14 after plating). $A, C=P_{1} ; B, D=P_{2.5} . A, B=$ Acute exposure assay, Schedule 1; C, D = late repeated exposure assay, Schedule 2 . Dashed line = cell growth assay; data are expressed as cell growth $(\%$ vs solvent control) \pm SD. Straight line $=\mathrm{CTA}$; data are expressed as mean number of type III foci/plate \pm SD; ${ }^{*}, \mathrm{p}<0.05$; one-sided Dunnett test.

\subsection{Evaluation of the transforming potential \\ of $\mathrm{PM}_{1}$ and $\mathrm{PM}_{2.5}$}

The detailed results of the CTAs are reported in Table S3 ${ }^{1}$. Untreated cells (negative controls) and solvent controls did not show formation of type III foci. The positive control 3-MCA $(1 \mu \mathrm{g} / \mathrm{ml})$ induced a statistically significant increase in the number of transformed foci, thus ensuring the validity of the study (Tab. S3 ${ }^{1}$ ).

In the acute exposure assay with the autumn samples, the foci number increased linearly with the tested concentrations $(\mathrm{p}<0.05$, Dunnett test) (Fig. 4A,B). The late repeated exposure assay was conducted only for the $\mathrm{PM}_{2.5}$ autumn extract. A significant and concentration-dependent increase of transformed foci was induced at concentrations of 4,6 , and $8 \mathrm{~m}^{3} /$ well $(\mathrm{p}<0.05$, Dunnett test) (Fig. 4D).

In the acute exposure assay performed with the summer extracts, no statistically significant increase of transformed foci was observed (Fig. 4A,B). In the late repeated exposure assay, the number of transformed foci increased concentration-dependently after repeated treatment with both $\mathrm{PM}_{1}$ and $\mathrm{PM}_{2.5}$ summer extracts at doses 4,6 , and $8 \mathrm{~m}^{3} /$ well $(\mathrm{p}<0.05)$ (Fig. 3C,D).
The increase in cell transformation induced by the autumn PM extracts was directly related to the amount of PM collected during the sampling campaign (Fig. 5A,C). Moreover, the increase of transformed foci induced by the autumn samples was associated with the overall amount of PAHs and NPAHs ( $\Sigma$ PAHs $+\Sigma$ NPAHs) measured in the extracts (Fig. 5A,C).

When cells were treated with the summer samples according to the acute exposure schedule, concentrations of $\mathrm{PM}$ in either $\mathrm{PM}_{1}$ and $\mathrm{PM}_{2.5}$ up to $80 \mu \mathrm{g} /$ well did not induce cell transformation. However, the late repeated administration of the summer samples significantly enhanced the onset of type III foci (Fig. 5B,D) in both $\mathrm{PM}_{1}$ and $\mathrm{PM}_{2.5}$.

\section{Discussion}

For environmental monitoring, the evaluation of hazard and the prediction of the health risks associated with complex mixtures of pollutants usually rely on the measured concentrations of single components of the mixture, in order to ensure that the legal 


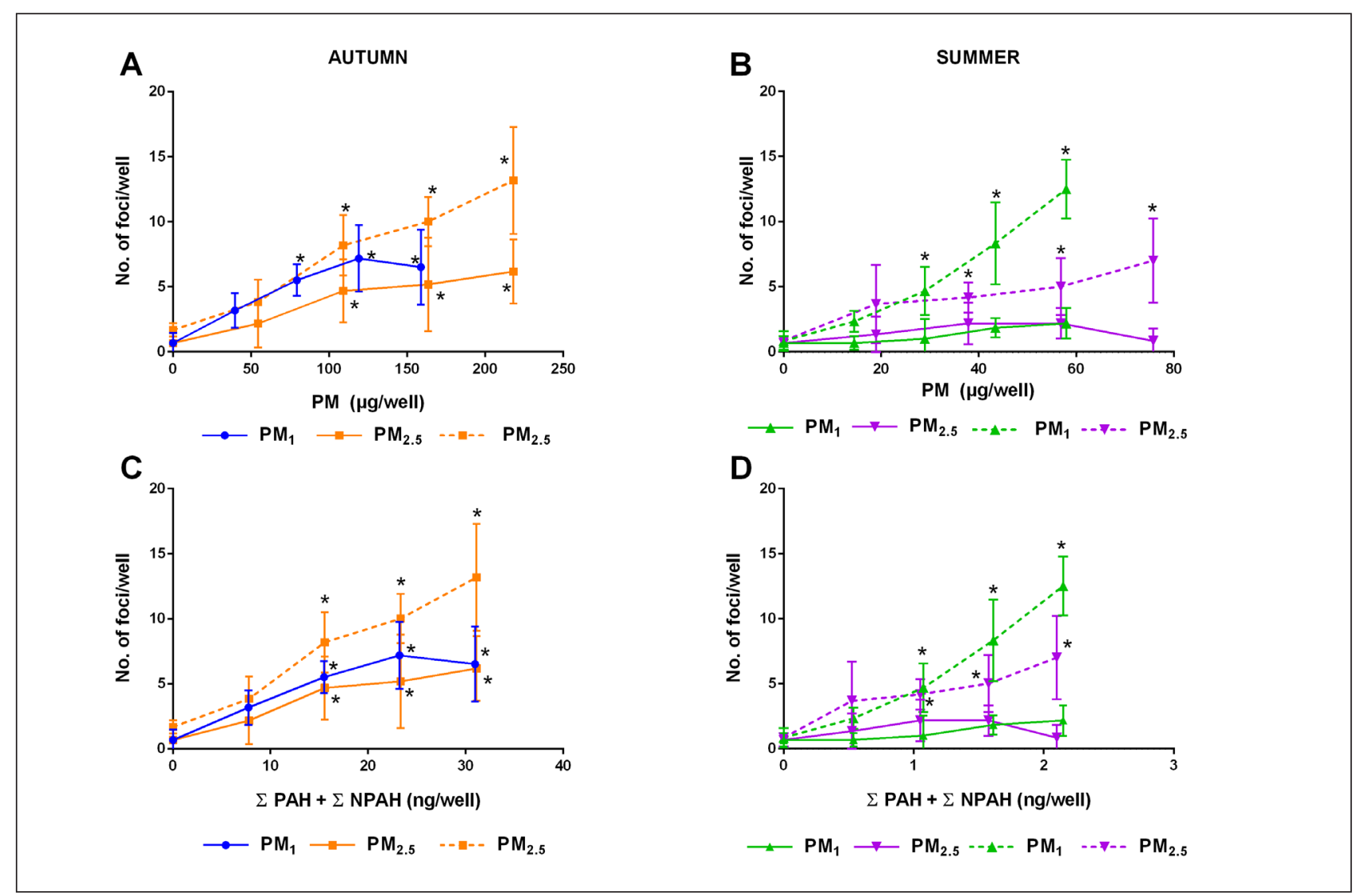

Fig. 5: Relationships between the number of type III foci and the total amount of PM or PAHs and NPAHs present in each treatment extract

$\mathrm{A}, \mathrm{B}=\mathrm{PM}(\mu \mathrm{g} / \mathrm{well}) ; \mathrm{C}, \mathrm{D}=\Sigma \mathrm{PAHs}+\Sigma \mathrm{NPAHs}$ (ng/well). Straight line = acute exposure assay, Schedule 1. Dashed line $=$ late repeated exposure assay, Schedule 2. Data are expressed as mean number of type III foci/plate $\pm S D$. *, $p<0.05$; one-sided Dunnett test

limit of each hazardous chemical is not exceeded. This approach has several limitations due to the presence in the mixtures of unknown chemicals and the lack of information concerning the possible interactions among components that may modify the toxicological outcome. In vivo testing of mixtures is not a feasible solution to address regulatory needs, not least due to the feasibility constraints to performing such resource-demanding assays for all possible mixture combinations.

The need to identify simple, fast, and robust hazard assessment tools for unknown pollutant matrices and for UVCBs (substances of unknown or variable composition, complex reaction products or biological materials) is acknowledged by regulatory authorities and industry. For example, in Europe UVCBs require regulation under REACH and Classification, Labeling and Packaging (CLP) Regulations by the European Chemicals Agency (ECHA). Therefore, the study reported here will have potential applications in characterizing the propensity of UVCBs to trigger promotion of carcinogenesis, and so can fill a key in vitro assay endpoint gap to provide carcinogenicity-relevant toxicological information regarding environmental pollutants and industrial chemical mixtures to support regulators in the decision-making process.

Most of the current validated in vitro assays are able to identify genotoxic chemicals but fail to recognize non-genotoxic carcinogenic compounds. Even though the CTA cannot be used as a stand-alone test for predicting carcinogenesis, it can address both genotoxic and some non-genotoxic carcinogenic modes of action. CTA models already have been successfully used to analyze complex mixtures and environmental contaminants (Vanparys et al., 2012; Corvi et al., 2012; Vasseur and Lasne, 2012)

In a previous study, we evaluated the transforming potential of $\mathrm{PM}_{2.5}$ by using the BALB/c 3T3 CTA in combination with transcriptomics (Vaccari et al., 2015). This approach allows the identification of pathway-based toxicity profiles, which are associated with exposure to $\mathrm{PM}_{2.5}$ organic extracts, providing mechanistic information and highlighting the early key events in the pathway that lead to malignant transformation. Indeed, analysis of the modulated KEGG pathways suggested the induction of early events in the multistep process, leading to cellular transformation, including p53 and MAPK signaling pathway and the T-cell 
receptor mediated pathway, all playing a central role in inflammation and apoptosis-mediated steps leading to cancer (Vaccari et al., 2015). However, the organic extracts of $\mathrm{PM}_{2.5}$ failed to induce cell transformation in the 3T3 CTA. The concentration of pollutants in the mixtures and the time of exposure were probably sufficient to highlight toxic effects, but not to achieve oncotransformation in the 3T3 model (Vaccari et al., 2015).

In this study, we applied an in vitro top-down approach using the Bhas 42 CTA to highlight the overall toxicity of the environmental samples as well as their ability to induce in vitro cell transformation. Airborne $\mathrm{PM}_{2.5}$ and $\mathrm{PM}_{1}$ samples were collected at a site that is regarded as representative of the urban background of a city located in the southeast of the Po valley in Italy. This region is considered one of the most polluted areas in the European Union, with peak levels during winter that often exceed European air quality standards. The high concentrations of PM are the result of the high level of anthropogenic emissions combined with stagnant meteorological conditions, such as thermal inversion, which typically occurs in the cold season and leads to the local accumulation of air pollutants.

The effects of PM samples were evaluated in both an acute and a repeated exposure schedule in the Bhas 42 CTA model. Transforming and cytotoxic properties were related to the higher content of PM in the autumn samples and strictly associated with the concentrations of PAHs (Fig. 3, 4). A clear concentration-response relationship was observed for both cytotoxic and transforming effects. However, it should be noted that, according to the validated protocol, the cytotoxicity assay was performed on proliferating cells in the acute exposure schedule and on quiescent cells in the late repeated exposure schedule. Cytotoxicity is an important parameter and confounder in the evaluation of the effects of chemicals in in vitro assays (Riss and Moravec, 2004; Judson et al., 2016; OECD, 2018) as well as in the possible mechanisms leading to cell transformation as a consequence of cell proliferation, in response to chemical toxicity (Jacobs et al., 2016). Therefore, the observed effects may be the result of a different response to the intrinsic toxicity of the treatment. No differences were observed that could be attributed to the different aerodynamic diameter of the collected PM.

The association between adverse health outcomes, including cancer, respiratory and cardiovascular diseases, and the exposure to complex mixtures containing pollutants, such as PAHs and NPAHs, has been deeply explored (IARC, 2010, 2013a,b; Pope et al., 2002; WHO, 2016). Several PAHs as well as PAH mixtures have been classified as possible or probable human carcinogens by IARC (IARC, 2010). Individual PAHs show a large variability in their carcinogenic potency. The co-presence of PAHs in binary chemical mixtures can result in more than additive effects at low and very low doses, whereas high doses exhibit an additive behavior (IARC, 2010).

The PM extracts were characterized with regard to their PAH and NPAH content (Tab. S2 ${ }^{1}$ ). They contained, among others, PAHs that are classified by IARC as Group 1 "Carcinogenic to humans" such as benzo[a]pyrene (IARC, 2012), as well as Group 2A "Probably carcinogenic to humans" such as cyclopenta[cd]pyrene, dibenz[a,h]anthracene, dibenzo[a,l]pyrene (IARC, 2010). Also, the nitro derivatives 6-nitrochrysene and 1-nitro- pyrene have been recently included in IARC Group 2A (IARC, 2013b). NPAHs were present in PM extracts at very low concentrations, often below the limit of detection. Only 1-nitropyrene was detected in the autumn PM samples. The samples were tested for mutagenicity and genotoxicity, giving inconclusive results, according to the UN Globally Harmonised System for classification and labelling of chemicals (GHS) criteria for mutagenicity classification (Bocchi et al., 2016; UN, 2017).

Here we have demonstrated that in the Bhas 42 CTA the transforming potential of the tested PM extracts is related to the overall amounts of PAHs and NPAHs ( 2 PAHs $+\Sigma$ NPAHs) that were measured in the extracts (Fig. 5). Several PAHs have been reported to be effective in the Bhas 42 CTA as single chemicals (Tab. S4 ${ }^{1}$ ) (Sakai et al., 2010, 2011; Tanaka et al., 2009; Asada et al., 2005; Misaki et al., 2016). Our results suggest that the adverse effects induced in Bhas 42 cells by a mixture containing PAHs could be the consequence of complex interactions among PAHs, duration of exposure, and variable activation of multiple mechanisms of action (Mascolo et al., 2018), which may be greater than the sum of the effects elicited by single chemicals. Indeed, the transforming properties of the tested samples were much higher than those of single chemicals. However, the possible presence of unidentified compounds, which could also contribute to the overall transforming activity of the analyzed mixtures, cannot be excluded.

In the experimental carcinogenesis models, the initiation/promotion process is conventionally represented by the application of subtransforming concentrations of a genotoxic chemical that are able to initiate the cells but not to sustain the tumor growth, followed by the chronic administration of a non-genotoxic chemical to promote the proliferation of initiated cells. In the Bhas 42 CTA protocol, only the promoting effects are highlighted by the chronic application of the tested chemical. This treatment would be sufficient to yield the foci formation from initiated cells in the cell population. Our results appear to provide confirmatory evidence regarding the initiated state of Bhas 42 cells (Sasaki et al., 2015). Indeed, the number of malignant foci at each concentration in the late repeated treatment schedule was double the number of foci obtained by treating the cells with the autumn samples according to the acute exposure protocol, suggesting that the prolonged treatment is able to select a significant number of already initiated cells, promoting the growth of malignant foci. The cellular transformation after a single chemical treatment in the acute exposure schedule may be due to the presence of multiple copies of the v-Ha-ras gene, which makes Bhas 42 cells prone to acquire a fully malignant phenotype. It may also be responsible for the high sensitivity of this assay to the chemical treatment and the short latency period preceding the expression of the transformed phenotype (Sasaki et al., 2015; OECD, 2017).

The Bhas 42 cell line is directed toward transformation by the transfection of the v-Ha-ras oncogene into the parental cell line (Sasaki et al., 1988, 1990b). The Ras family comprises three distinct genes ( $N$-Ras, $\mathrm{K}$-Ras, and $H$-Ras) and four distinct proteins. Whilst the expression of Ras wild-type isoforms varies depending on the cell lineage, tissue, or developmental stage, all three isoforms are regularly expressed in mouse and human tissues (Castellano and Santos, 2011). Wild-type isoforms play a role in different functions in a complex network of signaling pathways 
that are subject to feedback regulation (Kano et al., 2016). The mutated forms of the Ras genes can easily transform cells in vivo and in vitro and have been associated with a large variety of tumors and developmental syndromes (Castellano and Santos, 2011). Indeed, both mutationally activated and wild-type isoforms are involved in the tumorigenic process, since they all participate in the regulation of cancer-related signaling pathways.

In vitro studies showed that the H-Ras oncogene exhibits consistently higher transforming ability than oncogenic K-Ras and $N$-Ras when transfected into fibroblasts (Cheng et al., 2011). Few or no morphological changes were observed in exponentially growing v-Ha-ras-expressing NIH 3T3 cells, which exhibited transformed morphology at high cell densities (Jacobsen et al., 2002). The activation of the DNA damage response (DDR), which includes DNA damage detection pathways, cell cycle checkpoints, and DNA damage repair mechanisms, has been frequently associated with oncogenic Ras expression. The mutant $K$-Ras-driven tumorigenesis was sustained by the modulation of the DDR induced by wild-type $H$ - and $N$-Ras (Grabocka et al., 2014).

Moreover, Ras amplification is now believed to play a key role in tumor progression. Indeed, the number of gene copies and the level of protein expression can make the difference between initiation and progression of the tumor (Zhou et al., 2016). In the Bhas 42 cells, the $v$-Ha-ras oncogene is integrated in the chromosomal DNA (chromosome 17 and 19, 2.4 average copies/nucleus) (Sasaki et al., 2015) and this may influence downstream pathways, leading to events that have not been elucidated yet. Therefore, the Bhas 42 CTA may represent a good model to study the later steps of the initiation phase of the in vitro transformation of cells, whilst other CTA models, such as the BALB/c 3T3 model, may be more useful to highlight the early steps that sustain the cell transformation. Indeed, when samples from the same autumn campaign were analyzed in the BALB/c 3T3 CTA, under an experimental protocol similar to that of the acute exposure protocol in the Bhas 42 CTA, no malignant transformation of cells was observed up to the highest tested concentration $\left(12 \mathrm{~m}^{3}\right.$ equivalents/ plate) (Colacci et al., 2014).

Whilst the results reported here support the utility of the in vitro top-down approach to characterize the toxicity of real-life exposure mixtures, they also identify the need to select the appropriate testing strategy when in vitro tests are used as part of a test battery to contribute to the weight-of-evidence required to address complex adverse outcomes, such as carcinogenesis. This is particularly true in the regulatory context, when the results contribute to the policy decision process. In this context, it would be important to highlight early events, which may be used to support health prevention policies, while models resembling the late steps of cancer progression may be useful to understand the risks for susceptible groups of the population or the risk associated with the presence of mutated key genes, such as in hereditary FAP disease and sporadic colon cancer. As reported before, the timing of a Ras mutation in the sequence of key events leading to colon cancer is critical to define the likelihood of cancer progression (Raskov et al., 2014).

The identification of the mechanism and mode of action of chemicals leading to the adverse outcome should be part of any in vitro testing strategy to better support the biological plausibil- ity of the experimental results. The integrative use of in vitro assays targeting different endpoints, such as morphological transformation, also combined with omics-based approaches (Mascolo et al., 2018), could further help to enhance the accuracy of the evaluation of the carcinogenic potential of chemical mixtures, ultimately leading to the development of more targeted strategies in the mitigation and regulation of environmental airborne pollution.

\section{References}

Asada, S., Sasaki, K., Tanaka, N. et al. (2005). Detection of initiating as well as promoting activity of chemicals by a novel cell transformation assay using v-Ha-ras-transfected BALB/c 3 T3 cells (Bhas 42 cells). Mutat Res 588, 7-21. doi:10.1016/j. mrgentox.2005.07.011

Bocchi, C., Bazzini, C., Fontana, F. et al. (2016). Characterization of urban aerosol: Seasonal variation of mutagenicity and genotoxicity of $\mathrm{PM}_{2.5}, \mathrm{PM}_{1}$ and semi-volatile organic compounds. Mutat Res 809, 16-23. doi:10.1016/j.mrgentox. 2016.07.007

Castellano, E. and Santos, E. (2011). Functional specificity of Ras isoforms: So similar but so different. Genes Cancer 2, 216-231. doi:10.1177/1947601911408081

Cheng, C. M., Li, H., Gasman, S. et al. (2011). Compartmentalized Ras proteins transform NIH 3T3 cells with different efficiencies. Mol Cell Biol 31, 983-997. doi:10.1128/MCB. 00137-10

Colacci, A., Vaccari, M., Mascolo, M. et al. (2014). Alternative testing methods for predicting health risk from environmental exposures. Sustainability 6, 5265-5283. doi:10.3390/ su6085265

Colacci, A. and Vaccari, M. (2017). Children's and adult involuntary and occupational exposures and cancer. In M. Waters and C. Hughes (eds.), Translational Toxicology and Therapeutics: Windows of Developmental Susceptibility in Reproduction and Cancer. Hoboken, NJ, USA: John Wiley \& Sons, Inc. doi:10.1002/9781119023647.ch8

Corvi, R. and Vanparys, P. (2012). International prevalidation study on cell transformation assay. Preface. Mutat Res 744, 1-2. doi:10.1016/j.mrgentox.2012.02.004

Corvi, R., Aardema, M. J., Gribaldo, L. et al. (2012). ECVAM prevalidation study on in vitro cell transformation assays: General outline and conclusions of the study. Mutat Res 744, 12-19. doi:10.1016/j.mrgentox.2011.11.009

Corvi, R., Madia, F., Guyton, K. Z. et al. (2017). Moving forward in carcinogenicity assessment: Report of an EURL ECVAM/ ESTIV workshop. Toxicol In Vitro 45, 278-286. doi:10.1016/j. tiv.2017.09.010

EU (2008). Council Regulation (EC) No 440/2008 of 30 May 2008 laying down test methods pursuant to Regulation (EC) No 1907/2006 of the European Parliament and of the Council on the Registration, Evaluation, Authorisation and Restriction of Chemicals (REACH). OJ L142, 1-739. http://data.europa. eu/eli/reg/2008/440/oj

Gharibvand, L., Shavlik, D., Ghamsary, M. et al. (2017). The association between ambient fine particulate air pollution and 
lung cancer incidence: Results from the AHSMOG-2 study. Environ Health Perspect 125, 378-384. doi:10.1289/EHP124

Goodson, W. H., Lowe, L., Carpenter, D. O. et al. (2015). Assessing the carcinogenic potential of low-dose exposures to chemical mixtures in the environment: The challenge ahead. Carcinogenesis 36, Suppl 1, S254-296. doi:10.1093/carcin/bgv039

Grabocka, E., Pylayeva-Gupta, Y., Jones, M. J. et al. (2014). Wild-type $\mathrm{H}$ - and N-Ras promote mutant K-Ras-driven tumorigenesis by modulating the DNA damage response. Cancer Cell 25, 243-256. doi:10.1016/j.ccr.2014.01.005

Hamra, G. B., Guha, N., Cohen, A. et al. (2014). Outdoor particulate matter exposure and lung cancer: A systematic review and meta-analysis. Environ Health Perspect 122, 906-911. doi:10.1289/ehp.1408092

Han, S. G., Pant, K., Bruce, S. W. and Gairola, C. G. (2016). Bhas 42 cell transformation activity of cigarette smoke condensate is modulated by selenium and arsenic. Environ $\mathrm{Mol}$ Mutagen 57, 220-228. doi:10.1002/em.22000

IARC (2010). Some non-heterocyclic polycyclic aromatic hydrocarbons and some related exposure. IARC Monographs on the Evaluation of Carcinogenic Risks to Humans 92, 1-853. https://monographs.iarc.fr/wp-content/uploads/2018/06/ mono92.pdf

IARC (2012). Chemical agents and related occupations: A review of human carcinogens. IARC Monographs on the Evaluation of Carcinogenic Risk to Humans 100F, 1-599. https:// monographs.iarc.fr/wp-content/uploads/2018/06/mono100F. pdf

IARC (2013a). Air pollution and cancer. IARC Scientific Publication 161, 1-169. https://www.iarc.fr/news-events/iarcscientific-publication-no-161-air-pollution-and-cancer-asan-e-book/

IARC (2013b). Diesel and gasoline engine exhausts and some nitroarenes. IARC Monographs on the Evaluation of Carcinogenic Risks to Humans 105, 1-703. https://monographs.iarc.fr/ wp-content/uploads/2018/06/mono105.pdf

IARC (2016). Outdoor air pollution. IARC Monographs on the Evaluation of Carcinogenic Risks to Humans 109, 1-448. https:// publications.iarc.fr/538

Jacobs, M. N., Colacci, A., Louekari, K. et al. (2016). International regulatory needs for development of an IATA for non-genotoxic carcinogenic chemical substances. ALTEX 33, 359-392. doi:10.14573/altex.1601201

Jacobsen, K., Groth, A. and Willumsen, B. M. (2002). Ras-inducible immortalized fibroblasts: Focus formation without cell cycle deregulation. Oncogene 21, 3058-3067. doi:10.1038/ sj.onc. 1205423

Judson, R., Houck, K., Martin, M. et al. (2016). Analysis of the effects of cell stress and cytotoxicity on in vitro assay activity across a diverse chemical and assay space. Toxicol Sci 153, 409. doi:10.1093/toxsci/kfw148

Kakunaga, T. and Crow, J. D. (1980). Cell variants showing differential susceptibility to ultraviolet light-induced transformation. Science 209, 505-507. doi:10.1126/science.7394516

Kano, Y., Cook, J. D., Lee, J. E. and Ohh, M. (2016). New structural and functional insight into the regulation of Ras. Semin Cell Dev Biol 58, 70-78. doi:10.1016/j.semcdb.2016.06.006
Landrigan, P. J., Fuller, R., Acosta, N. J. R. et al. (2018). The Lancet Commission on pollution and health. Lancet 391, 462512. doi:10.1016/s0140-6736(17)32345-0

Lilienblum, W., Dekant, W., Foth, H. et al. (2008). Alternative methods to safety studies in experimental animals: Role in the risk assessment of chemicals under the new European chemicals legislation (REACH). Arch Toxicol 82, 211-236. doi:10.1007/s00204-008-0279-9

Lo, K. Y. and Kakunaga, T. (1982). Similarities in the formation and removal of covalent DNA adducts in benzo(a) pyrene-treated BALB/3T3 variant cells with different induced transformation frequencies. Cancer Res 42, 2644-2650.

Loomis, D., Grosse, Y., Lauby-Secretan, B. et al. (2013). The carcinogenicity of outdoor air pollution. Lancet Oncol 14, 12621263. doi:10.1016/S1470-2045(13)70487-X

Mascolo, M. G., Perdichizzi, S., Rotondo, F. et al. (2010). BALB/c 3T3 cell transformation assay for the prediction of carcinogenic potential of chemicals and environmental mixtures. Toxicol In Vitro 24, 1292-1300. doi:10.1016/j.tiv.2010.03.003

Mascolo, M. G., Perdichizzi, S., Vaccari, M. et al. (2018). The transformics assay: First steps for the development of an integrated approach to investigate the malignant cell transformation in vitro. Carcinogenesis 39, 955-967. doi:10.1093/carcin/ bgy037

Misaki, K., Takamura-Enya, T., Ogawa, H. et al. (2016). Tumour-promoting activity of polycyclic aromatic hydrocarbons and their oxygenated or nitrated derivatives. Mutagenesis 31, 205-213. doi:10.1093/mutage/gev076

OECD (2017). Guidance Document on the In Vitro Bhas 42 Cell Transformation Assay. OECD Series on Testing and Assessment 231. http://www.oecd.org/officialdocuments/pub licdisplay documentpdf/?cote $=\mathrm{ENV} / \mathrm{JM} / \mathrm{MONO}(2016) 1 \& \mathrm{doc}$ Language $=$ En

OECD (2018). Guidance Document on Good In Vitro Method Practices (GIVIMP). OECD Series on Testing and Assessment 286. OECD Publishing, Paris. doi:10.1787/97892643047961 -en

Ohmori, K., Sato, Y., Nakajima, D. et al. (2013). Characteristics of the transformation frequency at the tumor promotion stage of airborne particulate and gaseous matter at ten sites in Japan.Environ Sci Process Impacts 15, 1031-1040.doi:10.1039/ c3em00076a

Pope, C. A., $3^{\text {rd }}$, Burnett, R. T., Thun, M. J. et al. (2002). Lung cancer, cardiopulmonary mortality, and long-term exposure to fine particulate air pollution. JAMA 287, 1132-1141. doi:10.1001/jama.287.9.1132

Raskov, H., Pommergaard, H. C., Burcharth, J. and Rosenberg, J. (2014). Colorectal carcinogenesis - Update and perspectives. World J Gastroenterol 20, 18151-18164. doi:10.3748/ wjg.v20.i48.18151

Riss, T. L. and Moravec, R. A. (2004). Use of multiple assay endpoints to investigate the effects of incubation time, dose of toxin, and plating density in cell-based cytotoxicity assays. Assay Drug Dev Technol 2, 51-62. doi:10.1089/ 154065804322966315

Sakai, A., Sasaki, K., Muramatsu, D. et al. (2010). A Bhas 42 cell transformation assay on 98 chemicals: The characteristics and 
performance for the prediction of chemical carcinogenicity. Mutat Res 702, 100-122. doi:10.1016/j.mrgentox.2010.07.007

Sakai, A., Sasaki, K., Hayashi, K. et al. (2011). An international validation study of a Bhas 42 cell transformation assay for the prediction of chemical carcinogenicity. Mutat Res 725, 57-77. doi:10.1016/j.mrgentox.2011.07.006

Sasaki, K., Mizusawa, H. and Ishidate, M. (1988). Isolation and characterization of ras-transfected BALB/3T3 clone showing morphological transformation by 12-O-tetradecanoylphorbol-13-acetate. Jpn J Cancer Res 79, 921-930. doi:10.1111 jj.1349-7006.1988.tb00056.x

Sasaki, K., Mizusawa, H., Ishidate, M. and Tanaka, N. (1990a). Transformation of ras transfected BALB 3T3 clone (Bhas 42) by promoters: Application for screening and specificity of promoters. Toxicol In Vitro 4, 657-659. doi:10.1016/08872333(90)90137-i

Sasaki, K., Mizusawa, H., Ishidate, M. et al. (1990b). Establishment of a highly reproducible transformation assay of a ras-transfected BALB 3 T3 clone by treatment with promoters. Basic Life Sci 52, 411-416. doi:10.1007/978-1-4615-9561$8 \_42$

Sasaki, K., Umeda, M., Sakai, A. et al. (2015). Transformation assay in Bhas 42 cells: A model using initiated cells to study mechanisms of carcinogenesis and predict carcinogenic potential of chemicals. J Environ Sci Health C Environ Carcinog Ecotoxicol Rev 33, 1-35. doi:10.1080/10590501.2014.967058

Tanaka, N., Sasaki, K., Hayashi, K. et al. (2009). An interlaboratory collaborative study on a cell transformation assay using Bhas 42 cells. AATEX 14, 831-848. doi:10.11232/aatex.14.831

Uchio-Yamada, K., Kasai, F., Ozawa, M. and Kohara, A. (2017). Incorrect strain information for mouse cell lines: Sequential influence of misidentification on sublines. In Vitro Cell Dev Biol Anim 53, 225-230. doi:10.1007/s11626-016-0104-3

UN (2017). Globally Harmonized System of Classification and Labelling of Chemicals (GHS): Seventh revised edition. New York, USA: United Nation. doi:10.18356/e9e7b6dc-en

US-EPA (2011). Exposure factors handbook: 2011 edition. U.S. Environmental Protection Agency, National Center for Environmental Assessment. EPA/600/R-09/052F. S. National Technical Information Service, VA, i-lxii.

Vaccari, M., Mascolo, M. G., Rotondo, F. et al. (2015). Identifica- tion of pathway-based toxicity in the BALB/c 3 T3 cell model. Toxicol In Vitro 29, 1240-1253. doi:10.1016/j.tiv.2014.10.002

Vanparys, P., Corvi, R., Aardema, M. et al. (2011). ECVAM prevalidation of three cell transformation assays. ALTEX 28, 56-59. doi:10.14573/altex.2011.1.056

Vanparys, P., Corvi, R., Aardema, M. J. et al. (2012). Application of in vitro cell transformation assays in regulatory toxicology for pharmaceuticals, chemicals, food products and cosmetics. Mutat Res 744, 111-116. doi:10.1016/j.mrgentox.2012.02.001

Vasseur, P. and Lasne, C. (2012). OECD detailed review paper (DRP) number 31 on "cell transformation assays for detection of chemical carcinogens": Main results and conclusions. $\mathrm{Mu}$ tat Res 744, 8-11. doi:10.1016/j.mrgentox.2011.11.007

Weisensee, D., Poth, A., Roemer, E. et al. (2013). Cigarette smoke-induced morphological transformation of Bhas 42 cells in vitro. Altern Lab Anim 41, 181-189. doi:10. 1177/026119291304100207

WHO (2016). Ambient Air Pollution: A Global Assessment of Exposure and Burden of Disease. Geneva, Switzerland: World Health Organization. https://apps.who.int/iris/handle/ 10665/250141

Zhou, B., Der, C. J. and Cox, A. D. (2016). The role of wild type RAS isoforms in cancer. Semin Cell Dev Biol 58, 60-69. doi:10.1016/j.semcdb.2016.07.012

\section{Conflict of interest}

The authors declare that they have no potential or actual conflicts of interest.

\section{Acknowledgments}

This work has been entirely supported by Regione Emilia Romagna - Supersite Project (Act 428/08.02.2010). We would like to thank the Environmental Mutagenesis Thematic Laboratory Regional Agency for Prevention, Environment and Energy Emilia Romagna (Arpae) for the preparation of the PM extracts and the Polo Analitico Regionale Microinquinanti Organici - Arpae for performing the chemical characterization of the PM extracts. We also thank Wolfgang Voelkner for providing the facilities and the equipment for the Bhas 42 experimental work as well as scientific guidance and helpful discussions. 